\title{
Hybrid ARQ Scheme Using Complementary Punctured Turbo (CPT) Codes
}

\author{
Yang Zhang ${ }^{1}$, Fan Yang ${ }^{1, *}$, Zhongtao Huang ${ }^{2}$, Weitong Zhao ${ }^{2}$ and Keyu Long ${ }^{2}$ \\ ${ }^{1}$ School of Communication and Information Engineering, University of Electronic Science and Technology of China, Chengdu \\ 611731, China \\ ${ }^{2}$ The Second Research Institute of CAAC, Chengdu 610041, China \\ ${ }^{*}$ Corresponding author
}

\begin{abstract}
We propose a hybrid automatic repeat request (HARQ) scheme by using complementary punctured turbo (CPT) codes. The information bit sequence is encoded by a rate- $1 / 3$ convolutional turbo code, and then punctured by different sets of coded bits using complementary perforation patterns, which is used for turbo decoding. In our proposed HARQ process, the sets of coded bits are sent alternatively, and are combined with previous stored code sequence at the receiver. Using the same channel conditions, the conducted simulation results show that our proposed scheme is superior over the three well known HARQ schemes for a wide range of signal-to-noise ratios (SNRs) in terms of the average transmit power-saving and system throughput.
\end{abstract}

Keywords-Hybrid ARQ; throughput; turbo codes; iterative decoding; complementary punctured; recursive convolutional codes

\section{INTRODUCTION}

Hybrid automatic repeat request (HARQ) system, which is a combination of automatic repeat request (ARQ) and forward error correction (FEC), has been proved to be an important error control technique in communications systems. In standard ARQ scheme, an error-detecting code such as cyclic redundancy (CRC) code is used for packet checking, while in HARQ scheme, a forward error-correcting code such as turbo code $^{[1]}$ adopted in 3GPP LTE standard in addition to the errordetecting code is employed. The HARQ scheme at the expense of more channel capacity has better performance than the standard ARQ especially in poor channel conditions, however, the ARQ scheme added few redundancy bits for error-detecting can potentially reach high throughput in good channel conditions. As a result, there exists a crossing point in terms of throughput versus SNR, i.e., the HARQ scheme is better below this point, and the standard ARQ scheme is more efficient above this point. The crossing point also exits in different HARQ schemes. Because the error correction capability of FEC codes differs from one another, HARQ schemes use different FEC codes, even using the same code with inconsistent retransmission order, which will result in different throughput versus SNR curves and a crossing point therein. In the following, we consider two typical HARQ schemes which are turbo-principle HARQ ${ }^{[2]}$ and twin-component turbo (TCT) $\mathrm{HARQ}^{[3]}$.

Another important question is: How can we implement a HARQ scheme that is optimal in terms of throughput versus SNR for the whole SNR range considered, while keeping relatively low transmit power and computational complexity.
There are a few literatures which well balance the two above aspects. In [4], the authors improved the throughput of TCT HARQ by using multi-level turbo codes, however, it needs additional interleavers in hardware implementation. On the other hand, the authors of [5] explored a new early stopping approach for efficient turbo iteration without degrading the throughput of TCT HARQ. Further, the authors of [6] proposed a deferred iterations strategy for complexity reduction in prevalent channel conditions.

We take above two aspects into consideration. Firstly, we use soft combining technique rather than using log-likelihood ratios (LLRs) as the a priori information. Chase combining (CC) and incremental redundancy (IR) are two main soft combining techniques in HARQ schemes. For chase combining, the same coded bits are retransmitted and combined with previous received transmissions using diversity combining. For incremental redundancy, different sets of the coded bits are generated by puncturing the encoder output. Then the retransmission is carried out by using different coded bits from the previous one. Secondly, we apply turbo codes with complementary structure for a simple ARQ mechanism in each transmission. Motivated by complementary punctured convolutional (CPC) codes developed in [7], we propose a complementary punctured turbo (CPT) HARQ scheme. The rate-1/2 punctured turbo code and its complementary pattern are alternatively sent in every retransmission. Following CPC codes, the concept of the CPT codes is technically straightforward, however, there are several notable distinctions. On one hand, the convolutional encoder is replaced by a turbo encoder, and thus the Viterbi decoder is replaced with multiple component SISO decoders which are constitute as a turbo decoder. On the other hand, the CPC codes can be punctured in an arbitrary position, while only the parity-check bits in our proposed CPT codes are punctured.

For simplicity, a stop-and-wait ARQ protocol and BPSK modulation are specified in the following discussion. We assume that the return channel is perfect, i.e., there is no errors for sending an acknowledgment signal (ACK) from the receiver back to the transmitter.

\section{COMPLEMENTARY PUNCTURED TURBO CODES}

A perforation matrix (or pattern) $P$ of period $m$ applied to a turbo code having ${ }^{n}$ output sub-sequences can be represented by: 


$$
P=\left[\begin{array}{cccc}
\mathrm{g}_{11} & \mathrm{~g}_{12} & \ldots & \mathrm{g}_{1 \mathrm{~m}} \\
\mathrm{~g}_{21} & \mathrm{~g}_{22} & \ldots & \mathrm{g}_{2 \mathrm{~m}} \\
\ldots & \ldots & \ldots & \ldots \\
\mathrm{g}_{\mathrm{n} 1} & \mathrm{~g}_{\mathrm{n} 2} & \cdots & \mathrm{g}_{\mathrm{nm}}
\end{array}\right],
$$

where only the first row corresponds to the systematic subsequence, while other rows correspond to the parity-check subsequences. Notice that $\mathrm{g}_{i j} \in\{0,1\}$, where $1 \leq i \leq n$ and $1 \leq j \leq m$ denotes the puncturing position at $i$-th row and $j$-th column, and the value 0 implies that the corresponding bit is punctured.

Let $P_{k}, k=1,2, \ldots, K$, be the perforation matrix corresponding to code $\mathbf{c}_{k}$ of the same rate. According to [7], the $K$ codes are said to be complementary if every element in matrix $P$ is greater or equal than one, where $P$ is shown below:

$$
P=\sum_{k=1}^{K} P_{k}
$$

where a rate-1/3 turbo encoder with two sub-encoders (i.e., $\mathrm{k}=2$ ) is employed in our paper.

As shown in Figure 1, an information bit sequence $\mathbf{u}=\left[u_{1}, u_{2}, \ldots, u_{L}\right]$ is first encoded by $M$-bit CRC code, yielding a new sequence $\mathbf{v}=\left[v_{1}, v_{2}, \ldots, v_{N-\lambda}\right]$. Then $\mathbf{v}$ is further encoded by a rate- $1 / 3$ turbo code, where $\lambda$ is the memory of the component convolutional encoder, and $N=(L+M+\lambda)$.

Generally, the output binary sequence c from the encoder is punctured to ${ }^{\mathbf{c}_{1}}$ using $P_{l}$ and then multiplexes to $\hat{\mathbf{c}}_{1}$ using modulation mapping. For a complementary punctured turbo code, the sequence $\mathbf{c}_{2}$ is generated from $\mathbf{c}$ using $P_{2}$.

The codeword ${ }^{\mathrm{C}}$ can be divided into three sub-sequences, i.e., $\quad \mathbf{c}=\left[\mathbf{c}^{s} ; \mathbf{c}^{p 1} ; \mathbf{c}^{p^{2}}\right]$, where $\mathbf{c}^{s}=\left[c_{11}^{s}, c_{12}^{s}, \ldots, c_{1 N}^{s}\right], \quad \mathbf{c}^{p 1}=$ $\left[\mathrm{c}_{11}^{p 1}, \mathrm{c}_{12}^{p 1}, \ldots, \mathrm{c}_{1 N}^{p 1}\right]$, and $\mathbf{c}^{p 2}=\left[\mathrm{C}_{11}^{p 2}, \mathrm{c}_{12}^{p 2}, \ldots, \mathrm{C}_{1 N}^{p 2}\right]$ are the subsequences corresponding to the systematic sub-sequence, the first parity-check sub-sequence, and the second parity-check sub-sequence of the encoder outputs, respectively.

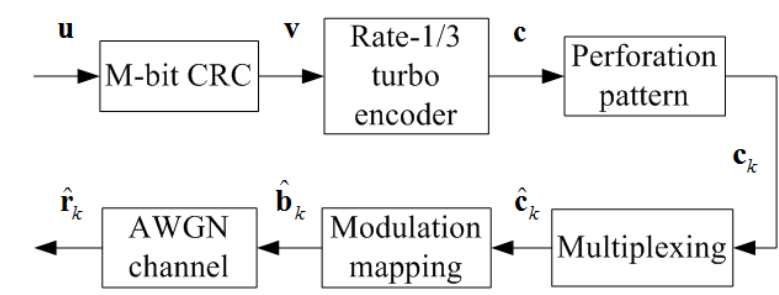

FIGURE I. THE TRANSMISSION USING A PUNCTURED TURBO CODE.

\section{OUR PROPOSED HARQ}

As indicated by [3], the throughput of the TCT HARQ scheme is larger than that of the Turbo-principle HARQ scheme[2] for a wide SNR range. However, there is performance loses for SNR interval between $1 \mathrm{~dB}$ and $4.5 \mathrm{~dB}$. Because the rate-1/2 turbo code used in [2] is more powerful than RCC in [3] in this SNR interval, which makes the Turboprinciple HARQ scheme able to decode the packet correctly during the first transmission most of the time. Motivated by this feature, we propose a Type-III HARQ scheme using complementary punctured turbo (CPT) codes, which is based on the complementary punctured convolutional (CPC) codes[7] The CPT HARQ scheme is the same as Turbo-principle HARQ scheme using rate- $1 / 2$ turbo code at the first transmission, while CPT HARQ scheme uses another rate- $1 / 2$ turbo code for the second transmission, where the two turbo codes are complementary in terms of their perforating parity-check bits. From the third transmission on, the CPT HARQ scheme alternatively uses the two kinds of turbo codes at the transmitter, and implements code combining at the receiver.

\section{A. The Encoder Structure}

The encoder procedure is shown in Figure 1. For the first transmission, a rate-1/2 turbo code $\mathbf{c}_{1}$ is generated by using perforation pattern ${ }^{P_{1}}$.Then the modulated sequence $\hat{\mathbf{b}}_{1}$ is sent to additive white Gaussian noise (AWGN) channel. If a NACK is received at the transmitter, a complementary rate- $1 / 2$ turbo code $\mathbf{c}_{2}$ is launched by using perforation pattern $P_{2}$, then a modulated sequence $\hat{\mathbf{b}}_{2}$ is transmitted, where $\hat{\mathbf{b}}_{2}=\left[b_{11}^{s}, b_{11}^{p 2}, b_{12}^{s}, b_{12}^{p 1}, \ldots, b_{1 N}^{s}, b_{1 N}^{p 1}\right]$ is generated from $\hat{\mathbf{c}}_{2}$ using modulation mapping. From the third transmission on, $\hat{\mathbf{b}}_{1}$ and $\hat{\mathbf{b}}_{2}$ are transmitted alternatively, i.e., $\hat{\mathbf{b}}_{1}$ is sent in odd transmission number, while $\hat{\mathbf{b}}_{2}$ is sent in even transmission number.

\section{B. The Decoder Structure}

Let $\hat{\mathbf{b}}^{(l)}$ be the output modulated sequence for the l-th transmission. In our proposed scheme, $\hat{\mathbf{b}}^{(l)}$ relative to the complementary transmitted sequences $\hat{\mathbf{b}}_{1}$ and $\hat{\mathbf{b}}_{2}$ is described as follows: 


$$
\hat{\mathbf{b}}^{(1)}=\left\{\begin{array}{l}
\hat{\mathbf{b}}_{1}, l=1,3,5, \ldots \\
\hat{\mathbf{b}}_{2}, l=2,4,6, \ldots
\end{array}\right.
$$

After passing through the AWGN channel, the received sequence $\hat{\mathbf{r}}^{(l)}$ is first demodulated to $\hat{\mathbf{z}}^{(l)}$, and then converted to $\mathbf{z}^{(l)}$ using de-multiplexing, where $\mathbf{z}^{(l)}$ is a sequence containing LLRs of each received bit. $\Lambda^{(l-1)}$ denotes the previous stored LLRs of each received bit, and $\Lambda^{(0)}$ is a zero vector. Whenever the packet is deemed to be in error and $l$ not exceeds the maximum retransmission number $\eta$, the receiver stores $\Lambda^{(l)}$ in the memory and sends a NACK back to the transmitter for retransmission. Considering $l>1$, prior to the regular turbo decoding, the receiver gets $\Lambda^{(l-1)}$ from memory and implements equal-gain diversity combining using $\mathbf{z}^{(l)}$ and $\Lambda^{(l-1)}$, yielding $\Lambda^{(0)}$, which is given by:

$$
\Lambda^{(l)}=\Lambda^{(l-1)}+\mathbf{z}^{(l)} .
$$

Notice that we can use other soft combining techniques instead of equal-gain diversity combining, but we adopt this simple combining technique for complexity consideration. The HARQ processing is ended if there is no detected error or $l$ is greater $\eta$.

The main advantage of our proposed Type-III HARQ scheme is that any complementary sequence sent each time can be decoded by itself. The decoder does not have to rely on previously received sequences for decoding the same data, which is desirable especially in situations where a transmitted packet can be lost or severely corrupted as a result of interference.

\section{Simulation Results}

We evaluate four HARQ schemes (including our proposed scheme) for average transmit power-saving, average decoding complexity, and throughput. We consider the rate-1/2 recursive systematic convolutional (RSC) code with generator polynomial $(13,15)$ in octal form, where ' 13 ' denotes the feedback part, and '15' represents the feed-forward part. This RSC code is adopted for TCT HARQ scheme[3] in the first transmission, which is also used as a component code in other three HARQ schemes involved by turbo codes.

The first component convolutional encoder of a turbo code is terminated using $\lambda$ bits, while the second component convolutional encoder is not. By using Log-MAP algorithm, the maximum number of turbo iterations $\xi=5$ is allowed for each transmission. The widely used 16-bit CRC-CCITT code with polynomial $\left(x^{16}+x^{12}+x^{5}+1\right)$ is applied to error detection. Because of the added additional redundant check bits, the four HARQ protocols provide throughput efficiencies a little less than $1 / 2$.

\section{A. The Computational Complexity}

Under the assumption that the same signal power is used for each transmission, Figure 2 shows the transmit powersaving evaluated by the average number of total transmission. On the other hand, Figure 3 derives the average number of turbo decoding iterations versus $E_{b} / N_{0}$ with the same simulation parameters, where $E_{b}$ denotes the energy per information bit and $N_{0}$ represents the power spectral density of the complex channel noise.

The following two figures illustrate that the simple turbo HARQ scheme needs much transmit power at the transmitter and the highest computational complexity in turbo decoding at the receiver. Because of involving turbo concept in ARQ procedure, the turbo-principle HARQ scheme is more efficient than the simple turbo HARQ, which has moderate consumption among the four HARQ schemes at both the transmitter and the receiver.

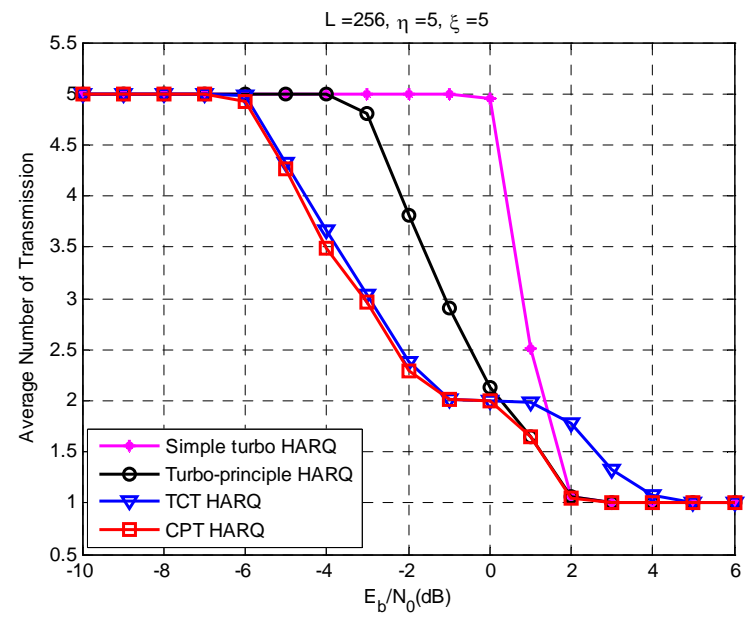

FIGURE II. THE COMPARISON OF THE AVERAGE NUMBER OF TOTAL TRANSMISSION FOR SENDING A PACKET

We consider the information packet $L=256$ for a maximum transmission $\eta=5$ and a maximum number of turbo iterations $\xi=5$. 


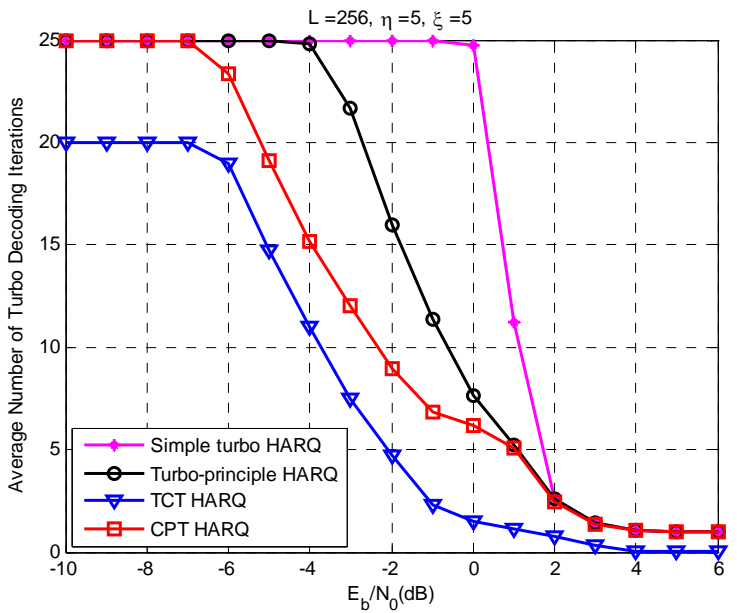

FIGURE III. THE AVERAGE NUMBER OF TURBO DECODING ITERATIONS USED IN THE FOUR HARQ SCHEMES

The simulation parameters are the same as in Figure 2.

By using a RCC encoder for the first transmission, the TCT HARQ has the lowest average number of turbo decoding iterations of the four schemes. However, the TCT HARQ scheme requires more transmit power than the other three schemes in SNR interval of between $1 \mathrm{~dB}$ and $4.5 \mathrm{~dB}$. The reason for this is that in this SNR interval, the turbo decoder can correctly derive the information bits most of time in the first transmission, while the RCC decoder can't. Usually, the TCT HARQ scheme should transmit the data packet more than once in this SNR interval and jointly decode the combined received data as a turbo decoder.

We can also observe from Figure 3 that the computational complexity of our proposed CPT HARQ scheme is a litter higher than that of the TCT HARQ scheme. however, our proposed CPT HARQ scheme has the minimum transmit power consumption among the four schemes for the whole SNR range considered.

\section{B. The Throughput Comparison}

In our simulations, the throughput efficiency is calculated according to the following:

$$
T_{h}=\frac{L}{2 N} \frac{N_{r}}{N_{t}},
$$

where $N_{r}$ and $N_{t}$ are the average number of data packets that are successfully received and the average number of transmission, respectively.

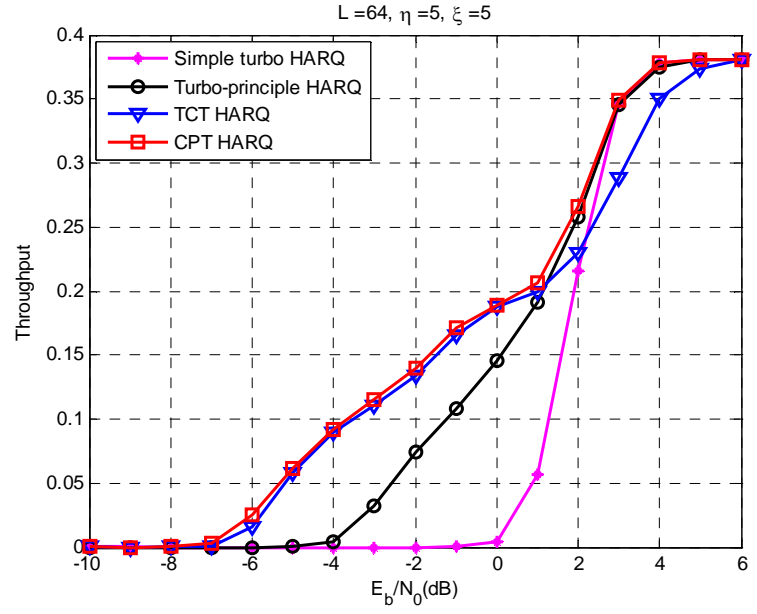

FIGURE IV. THROUGHPUT VERSUS $E_{b} / N_{0}$ OF THE FOUR HARQ

The information packet of size $L$ is set to 64 , and a maximum number of transmissions $\eta=5$ is allowed.

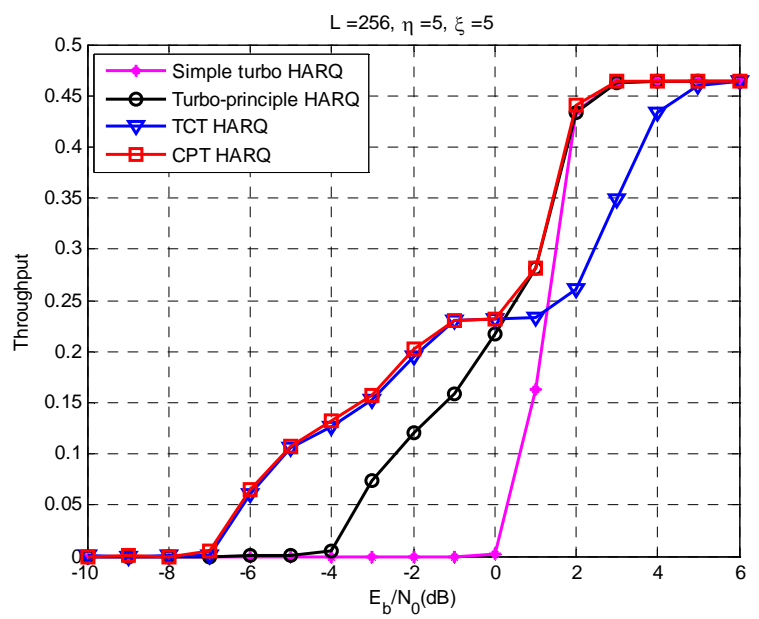

FIGURE V. THROUGHPUT VERSUS $E_{b} / N_{0}$ OF THE FOUR HARQ SCHEMES

The information packet of size $L$ is set to 256, and a maximum number of transmissions $\eta=5$ is allowed.

The throughput of the four HARQ schemes is evaluated by using different information packet sizes ( $L=64$ and $L=256$ ) for a fixed maximum number of transmission $\eta=5$. It can be observed from Figure 4 and Figure 5 that our proposed CPT HARQ scheme has almost the same throughput as the turboprinciple HARQ scheme for SNR in the range of $1 \mathrm{~dB}$ to $4.5 \mathrm{~dB}$, and our proposed scheme also has similar performance as TCT HARQ scheme for SNR below OdB. However, our proposed scheme outperforms all the other schemes in the whole SNR range considered. Moreover, it can be inferred that for SNR of range $1 \mathrm{~dB}$ to $4.5 \mathrm{~dB}$, the performance gap between the TCT 
HARQ scheme and any other schemes becomes wider as the information packet size increases, while for SNR below OdB,our proposed scheme and the TCT HARQ scheme are better than the single turbo HARQ scheme and the turboprinciple HARQ scheme, and the performance gain also increases along with the information packet size.

\section{CONCLUSIONS}

We proposed a Type-III HARQ scheme using complementary turbo codes. Our proposed CPT scheme can be implemented alternatively with a simple encoder and decoder structure. Moreover, the CPT scheme don't need no power control for doubling the transmit power, which losses the requirements for transmitter power amplifier. We evaluated the average transmit power-saving, the average number of turbo decoding iterations, and the throughput of four schemes. The conducted simulation results show that our proposed CPT HARQ scheme consumes moderate computational complexity for iterative decoding among the four schemes, while it has the minimum average transmit power and the highest throughput in the whole SNR range considered.

\section{ACKNOWLEDGMENT}

This work was supported in part by the National Natural Science Foundation of China (No. 61301272, No. 61675040, No. U1633129), and the Applied Basic Research Foundation of Science and Technology Department of Sichuan Province (No. 2014JY0037).

\section{REFERENCES}

[1] C. Berrou and A. Glavieux, "Near optimum error correcting coding and decoding: Turbo-codes," IEEE Trans. Commun., vol. 44, no. 10, pp. 1261-1271, Oct. 1996.

[2] K. R. Narayanan and G. L. Stu"ber, "A Novel ARQ Technique using the Turbo Coding Principle,” IEEE Commun. Lett., vol. 1, no. 2, pp. 4951,Mar. 1997.

[3] R. D. Souza, M. E. Pellenz, and T. Rodrigues, "Hybrid ARQ Schem Based on Recursive Convolutional Codes and Turbo Decoding," IEEE Trans. Commun., vol. 57, no. 2, pp. 315-318, Feb. 2009.

[4] H. Chen, R. G. Maunder, and L. Hanzo, "Multi-Level Turbo Decoding Assisted Soft Combining Aided Hybrid ARQ," IEEE VTC Spring, pp.15,2010 .

[5] "Low-Complexity Multiple-Component Turbo-Decoding-Aided Hybrid ARQ," IEEE Trans. Veh. Technol., vol. 60, no. 4, pp. 1571-1577,May 2011.

[6] "Lookup-Table-Based Deferred-Iteration Aided Low-Complexity Turbo Hybrid ARQ," IEEE Trans. Veh. Technol., vol. 60, no. 7, pp. 30453053,Sept. 2011.

[7] S. Kallel, "Complementary Punctured Convolutional (СРC) Codes and Their Applications," IEEE Trans. Commun., vol. 43, no. 6, pp. 20052009, June 1995 